www.jmscr.igmpublication.org

Impact Factor (SJIF): 6.379

Index Copernicus Value: 79.54

ISSN (e)-2347-176x ISSN (p) 2455-0450

crossrefDOI: https://dx.doi.org/10.18535/jmscr/v6i8.72

Journal Of Medical Science And Clinical Research

\title{
A Comparative Study of Lipid Profile and Atherogenic Index of Plasma among Women with Rheumatoid Arthritis and without Rheumatoid Arthritis
}

\author{
Authors \\ Dr Silpa Thota*, Dr Madhavi Kondeti, Dr Palla Nagadasaiah, Dr Pasupurekula Laxmi \\ Sri Venkateswara Medical College, Tirupati, Andhra Pradesh, India \\ *Corresponding Author \\ Dr Silpa Thota \\ Address: Flat no 403, Brindavanam Apartments, Thimminaidu Palem, Karkambadi Road, Tirupati \\ Andhra Pradesh 517507, India \\ Email: silpa.thota@yahoo.co.in, Phone NO-9493031608; 0877-2970021
}

\begin{abstract}
The clinical association between Rheumatoid arthritis $(R A)$ and cardiovascular diseases $(C V D)$ is important as the patients with rheumatoid arthritis are more prone to cardiovascular morbidity and mortality. Dyslipidemia appears to be present very early in the RA disease process which contributes to accelerated atherosclerosis among these individuals. Female gender is considered as risk factor for rheumatic diseases. So, this study aims at comparing the lipid profile levels and Atherogenic index of plasma (AIP) among women with $R A$ and women without $R A$. This observational study was conducted totally on 80 women in the age group of 30 to 60 years. Among them 40 women were clinically diagnosed with RA (as per 1987 ACR criteria) who are taken as group 1 and 40 women were without $R A$ who are taken as group 2. Serum lipid profiles parameters were estimated and Atherogenic index of plasma was calculated. These values were expressed in mean \pm standard deviation. The values are compared among both the groups and statistical significance was observed. Serum total cholesterol(TC), triglycerides(TAG), low density lipoprotein $(L D L)$, very low density lipoproteins(VLDL) and Atherogenic index of plasma were significantly increased in women with $R A$ and high density lipoprotein $(H D L)$ was significantly decreased in women with RA when compared with women without RA. Women with $R A$ are characterized by atherogenic lipid profile in comparison with women without RA. Early diagnosis and treatment of $R A$ along with screening of hyperlipidemias can reduce cardiovascular related mortality and morbidity in these patients.

Keywords: Atherogenic index of plasma; Cardiovascular risk; Lipid profile; Lipoproteins; Rheumatoid arthritis.
\end{abstract}

\section{Introduction}

Rheumatoid Arthritis (RA) is a chronic, systemic disease, in which various joints in the body are inflamed, leading to swelling, pain, stiffness, and the possible loss of function. It is an autoimmune disease in which the body's immune system attacks itself. Primarily, it is affecting the synovium leading to joint damage and bone 
destruction. ${ }^{(1)}$ It affects approximately 1 to $2 \%$ of general population worldwide and in India its incidence is $0.75 \%$. Female gender is a risk factor and RA is uncommon in men under the age of 45 where there is a 6:1 female excess. With increase in age there is increase in prevalence but sex difference of prevalence decreases. Onset is more during fourth and fifth decade of life. Prevalence of RA in India is much similar to that of prevalence in developed countries. $^{(2)}$

The disease usually presents as symmetric polyarthritis which leads to deformity in peripheral joint. The disease is not confined to joints, it progresses to become a systemic disease which involves many organs. Severe extra articular RA manifestations are associated with an increased risk of cardiovascular disease (CVD) events and are a major cause of cardiovascular morbidity in patients with RA. ${ }^{(3)}$

Studies have shown that in patients with RA, increased atherosclerosis leads to CVD which is the leading cause of premature mortality. The emergence of premature atherosclerosis is due to chronic inflammation in these patients. ${ }^{(4)-(5)}$

The pro-inflammatory cytokines, like interleukin 1 and 6, tumor necrosis factor alpha (TNF- $\alpha$ ), play a role in the development and progression of RA. These pro-inflammatory molecules and cytokines not only promote endothelial dysfunction and structural vessel abnormalities, but also induce other CV risk factors, such as changes in lipid levels, insulin resistance and oxidative stress. ${ }^{(6)}$

Atherogenic index of plasma (AIP) is the new marker of atherogenicity, since the AIP is related directly to the atherosclerosis significantly with increasing atherogenic risk (AIP from 0.24 to 0.51). ${ }^{(7)}$ Existence of hypertriglyceridemia will increase the activity of hepatic lipase which results in the increase of HDL-c catabolism (degradation of HDL-c). Degradation of one mg HDL-c will correlate with $2 \%$ increase in the risk coronary heart disease(8). AIP indicate a balance between the actual concentration of plasma total triglyceride and high density lipoprotein (HDL), which predetermine the direction of the cholesterol transport in an intravascular pool in the flux of newly produced cholesterol esters by lecithin cholesterol acyltransferase towards atherogenic LDLs beneficial HDLs ${ }^{(9)}$.

Women with RA have shown increase in mortality and morbidity when compared to general population. The main cause of mortality in these women is CVD. This study was designed to observe the lipid abnormalities and AIP in women with rheumatoid arthritis in comparison to women without RA.

\section{Materials and Methods}

This was an observational study from October 2015 to September 2016. A total of 80 women at age group of 30 to 60 years participated in the study. They are divided into 2 groups. Group 1 comprises of women who are proven cases of RA (as per 1987 ACR criteria) on the basis of detailed history and clinical examination including all investigations including radiographic and biochemical evaluation. Group 2 comprises of 40 women without RA who are considered as controls group.

Inclusion criteria: Women with rheumatoid arthritis in age group of 30 to 60yrs. The inclusion criteria for cases are based on Revised American Rheumatology association criteria (1987) for the diagnosis of rheumatoid arthritis. ${ }^{(10)}$

1) Morning stiffness in and around joints lasting at least 1 hour before maximal improvement.

2) Soft tissue swelling of three or more joint areas.

3) Swelling of proximal interphalangeal, metacarpo phalangeal and wrist joints.

4) Symmetrical swelling.

5) Rheumatoid nodules.

6) Positive rheumatoid factor.

7) Radiographic erosions or periarticular osteopenia in hand and /or wrist joints,

Criteria 1 to 4 must have been present for at least 6 weeks; RA is defined by the presence of 4 or more criteria. 
Exclusion criteria: Women with other form of arthritis, diabetes mellitus, hypertension, thyroid disorders, renal and liver disorders, hyperlipidemias treated with lipid lowering drugs were excluded from this study.

An informed written consent was taken from all the women who have participated and the study was well within the ethical norms. After making the subjects comfortable, under strict aseptic precautions $3 \mathrm{ml}$ of venous blood is collected after overnight fast in red topped tubes. Sample will be allowed to stand for 30 minutes and serum separated by centrifugation at $2000 \mathrm{rpm}$ for 10mins. Serum levels of Total cholesterol (TC), Triglycerides (TAG), High density lipoproteins (HDL) are measured immediately using commercially available Erba Mannheim kits by ERBA CHEM 7 semi autoanalyser.

Serum total cholesterol is estimated by Cholesterol oxidase peroxidise method (CHODPAP), Triglycerides are estimated by GPOTRINDER method, HDL cholesterol is estimated by phosphotungstic acid method, Low density lipoprotein (LDL) is calculated using friedwald formula, $\mathrm{LDL}-\mathrm{C}=\mathrm{TC}-\mathrm{TAG} / 5$ and very low density lipoprotein (VLDL) is calculated by using formula VLDL-C=TAG/5.

AIP was calculated as log of ratio (TG/ HDL-C) in $(\mathrm{mmol} / \mathrm{L})$. It has been suggested that AIP values of -0.3 to 0.1 are associated with low, 0.1 to 0.24 with medium and above 0.24 with high cardiovascular risk. $^{(11)}$

\section{Statistical analysis:}

The data collected was entered in excel sheet (Microsoft office excel 2007) .It is presented as mean \pm standard deviation and analysed using Fisher's exact test using SPSS Version 17.0 software. The "P" value of $<0.05$ was considered as statistically significant.

\section{Results}

In the present study the mean age for group 1 was $44.30 \pm 8.16$ and mean age for group 2 was 43.02 \pm 6.92 . Table 1 shows the mean values of lipid profile parameters and standard deviation along with statistical significance in women with RA and in women without RA. There was statistically significant raise in all lipid parameters except HDL-C in women with RA when compared with women without RA .There was statistically significant decrease in HDL-C level in women with RA when compared with women without RA. Table 2 shows AIP values in both the groups. The mean value and standard deviation of AIP in women with RA was significantly increased when compared to women without RA. Graph-1 shows comparison of mean lipid profile values $(\mathrm{mg} / \mathrm{dl})$ in group 1 and group 2.The comparison of mean AIP values of group 1 and group 2 are shown in Graph-2.

Table-1: Comparison of lipid profile parameters between group 1 and group 2

\begin{tabular}{|c|c|c|c|c|}
\hline \multirow{2}{*}{ Parameters } & Study group 1 & Study group 2 & \multirow{2}{*}{$P$ value } & \multirow{2}{*}{$\begin{array}{l}\text { Statistical } \\
\text { significance }\end{array}$} \\
\hline & Mean \pm S.D & Mean \pm S.D & & \\
\hline $\mathrm{TC}(\mathrm{mg} / \mathrm{dl})$ & $212.02+/-30.05$ & $190.65+/-38.08$ & $<0.01$ & $\mathrm{~S}$ \\
\hline TAG(mg/dl) & $208.83+/-64.62$ & $160.33+/-42.37$ & $<0.001$ & HS \\
\hline HDL-C(mg/dl) & $38.72+/-7.19$ & $49.04+/-6.53$ & $<0.001$ & HS \\
\hline VLDL-C (mg/dl) & $41.76+/-12.92$ & $32.06+/-8.47$ & $<0.001$ & $\mathrm{HS}$ \\
\hline $\begin{array}{l}\text { LDL-C }(\mathrm{mg} / \mathrm{dl}) \\
\text { AIP }\end{array}$ & $131.54+/-29.53$ & $109.54+/-39.72$ & $<0.01$ & $\mathrm{~S}$ \\
\hline
\end{tabular}

Table-2: Comparision of AIP values between group1 and group 2

\begin{tabular}{|l|c|c|c|}
\hline AIP value & Mean \pm S.D & P value & Statistical significance \\
\hline Group 1 & $0.35+/-0.17$ & $<0.001$ & HS \\
\hline Group 2 & $0.13+/-0.10$ & & \\
\hline
\end{tabular}

S: Significant; HS: Highly significant; NS: Not significant; S.D: standard deviation 
Graph-1: Comparison of mean lipid profile values (mg/dl) in both the groups

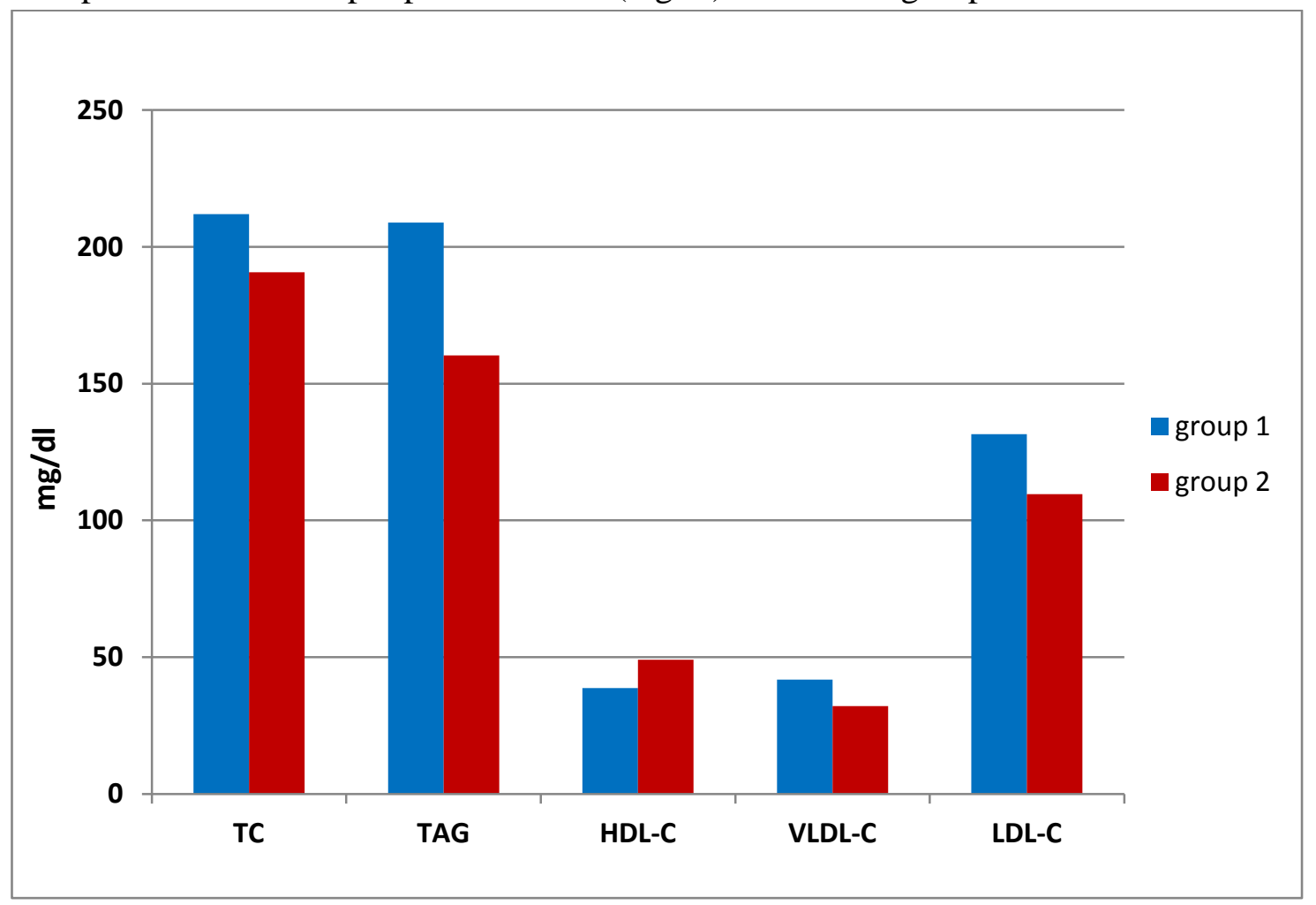

TC- Total cholesterol, TAG- Triacylglycerol, HDL-C- High density lipoprotein, VLDL-C- Very low density lipoprotein, LD-C - Low density lipo protein

Graph-2: Comparison of mean AIP values in both the groups

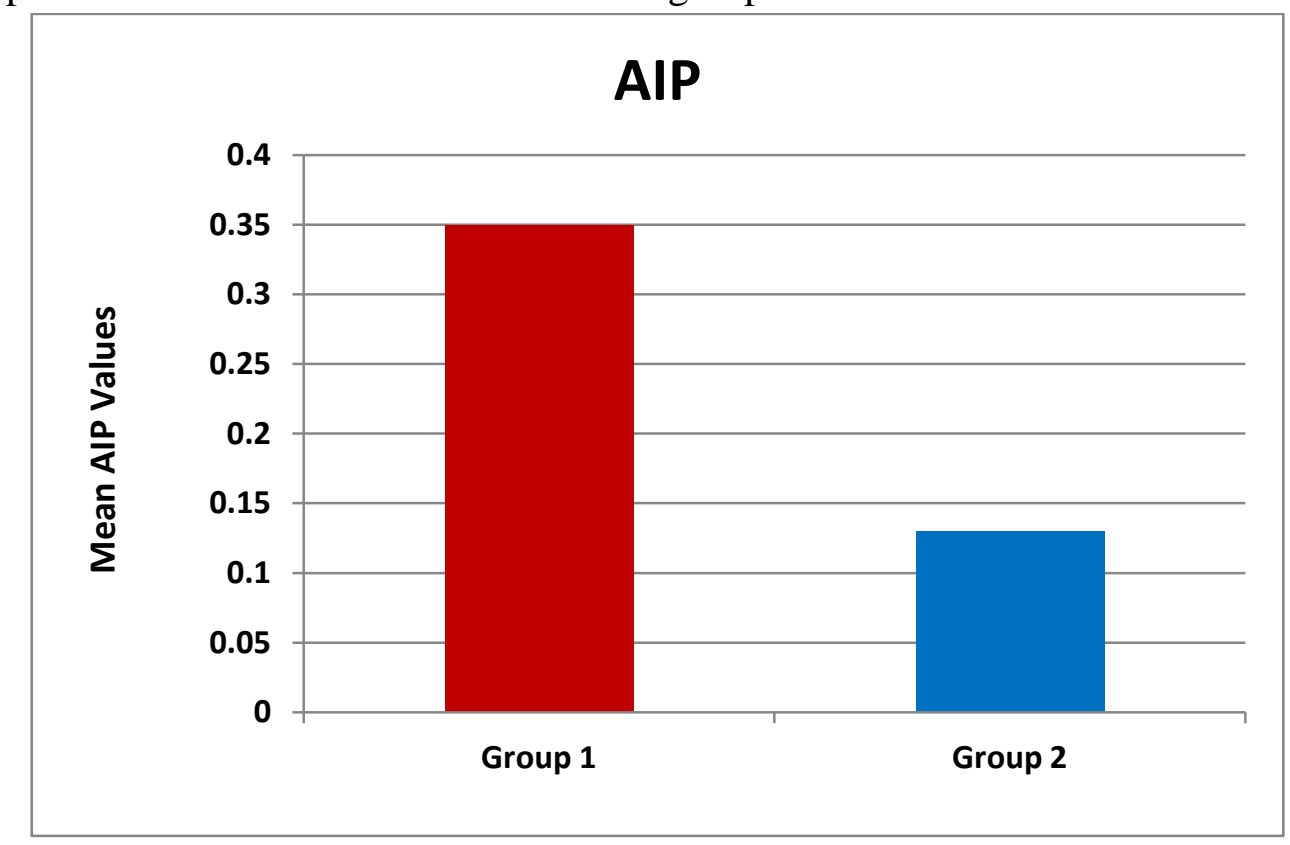

AIP- Atherogenic index of plasma

\section{Discussion}

In the present study we observed that there is dyslipidemia in women with RA. These women are found to be at increased risk for developing CVD, particularly coronary atherosclerosis. Our study showed that there is increased level of 
serum total cholesterol levels in women with RA. Hypercholestermia is an important factor in development of atherosclerosis. Low density lipoprotein has a positive relationship to the risk of coronary heart disease and also has been shown to undergo oxidation forming oxidized LDL. Oxidised LDL leads to phospholipid release, activating endothelial cells, thereby initiating an inflammatory process which leads to the formation of foam cells and subsequent fatty streaks. $^{(12)}$

We observed significantly lower levels of HDL-C in study group $(\mathrm{p}<0.001)$. Under normal condition HDL exerts its antiatherogenic role by protecting LDL from oxidation. These altered lipoproteins, along with an increase in lipid peroxidation products suggest that RA patients are at a high risk for the development of coronary heart disease. Some studies suggest that the decrease in HDL-C level may be due to reduced physical activity for the women with RA. Decreased HDL-C and increased LDL-C exposes women with RA to higher risk of atherosclerosis. ${ }^{(13)}$

In the present study the presence of high triglyceride levels in RA patients can be explained by the effects of cytokines at adipose tissue where it increases free fatty acid (FFA) release, at the liver it increases FFA and triglyceride synthesis and at the vascular endothelium where it reduces lipoprotein lipase activity.

Several studies have documented increased intima medial thickness which is a marker of early atherosclerosis and vascular risk in RA patients when compared to controls, suggesting a greater prevalence of sub clinical atherosclerosis in these patients. $^{(14)}$

In the present study the lipid profile parameters are significantly deranged in women with RA when compared with women without RA. Similarly in a study conducted by Athanasios N Georgiadis et $\mathrm{al}^{(15)}$, they observed that patients exhibited mild but significant higher baseline serum levels of TC, LDL-C, nonHDL-C, triglycerides and apoB where as HDL-C levels were significantly lower compared to controls and the study concluded that patients with early active RA before therapy has an atherogenic lipid profile. In another study conducted by Kowsalya $\mathrm{R}$ et $\mathrm{al}^{(16)}$ the study concluded that chronic systemic inflammation contributes significantly to excess cardiovascular disease in RA.

In contrast some studies where lipid profile of patients with RA has been evaluated, have reported lower levels of HDL-C and TC, higher serum concentrations of lipoprotein (a) and higher TC/HDL-C and LDL-C/HDL-C ratios in active and/or untreated disease than in the general population. However, other studies have not shown significantly different lipid levels from those observed in the healthy population and others refer to an overall reduction in all lipid subfractions in cases of active disease. These contrasting results could be attributed to the size of the samples, the type of study (prospective or cross-sectional), differences in the disease type (established or early), or to differences in the disease activity. Patients in remission or with controlled disease show an increase in HDL-C levels and a reduction in the atherogenic lipid profile when compared to patients with active disease. ${ }^{(17)-(19)}$

On evaluation of lipid ratios, in the current study, we observed that Atherogenic Index of Plasma (AIP) ratio was significantly higher in women with RA when compared to women without RA ( $p$ $<0.001$ ) The AIP ratio is 0.35 in group 1 and 0.13 in group 2 which are in accordance with suggested cut off, as there is increase in TAG values and decrease in HDL-C values. Similar finding of AIP was found in study done by Zeinab $\mathrm{M}$ A $\mathrm{R}$ et al.(20) Studies have shown that in situations where other atherogenic risk parameters like TG and HDLc appear normal, AIP may be the diagnostic alternative. Studies have shown its role in predicting cardiovascular risk. Thus, an increase in the atherogenic index in women with RA, suggesting that these patients are possibly exposed to a higher risk of atherosclerosis. 


\section{Conclusion}

Women with RA are characterised by dyslipidemias. Significant higher levels of TC, TAG, VLDL-C, LDL-C and AIP along with significant decrease in HDL-C suggesting that these women are possibly exposed to higher risk of atherosclerosis. As the inflammatory process of RA initiates cardiovascular damage, early diagnosis and treatment of RA along with screening of hyperlipidemias can reduce cardiovascular related mortality and morbidity in these patients. Early immuno-intervention for controlling inflammatory process and regular follow up of patient with estimation of lipid profile may reduce the risk of atherosclerosis and CVD in women with RA. Further studies are needed to estimate if early treatment helps these patients to reduce cardiovascular risk.

\section{Source of support: Nil}

\section{References}

1. Gravallese EM: Bone destruction in arthritis. Ann rheum Dis.2002, 61(Suppl2):ii84-ii86.

2. Dohert M, Lanyon P, Ralston SH. Inflammarotry Joint Disease.In:Christopher $H$, Edwin RC, Nicholas AB, Nicki RC, editors. Davidson "es Principles and Practice Of Medicine.19th ed.Edinburgh:Churchill Livingstone;2002.p.1002-07.

3. C Turesson, $\mathrm{R} \mathrm{L}$ McClelland, $\mathrm{T} \mathrm{J} \mathrm{H}$ Christianson, E L Matteson. Severe extra articular disease manifestations are associated with an increased risk of first ever cardiovascular events in patients with rheumatoid arthritis. Ann Rheum Dis. 2007; 66:70-75.

4. Rho YH, Chung CP, Oeser A, et al. Inflammatory mediators and premature coronary atherosclerosis in rheumatoid arthritis. Arthritis Rheum. 2009;61:1580-5.

5. Gerli R, Bocci EB, Sherer Y, et al. Association of anti-cyclic citrullinated peptide antibodies with subclinical atherosclerosis in patients with rheumatoid arthritis. Ann Rheum Dis. 2008;67:724-5.

6. LibbyP. Role of inflammation in atherosclerosis associated with rheumatoid arthritis, Am J Med, 2008, vol. 121 Suppl 1(pg. S21-31).

7. Susanti E. Donosepoetro M. Patellong I. Arif M. Differences between several atherogenic parameters in patients with controlled and uncontrolled type 2 diabetes mellitus. Med J Indones.2010;19:103-8.

8. Kavitha G. Ramani G. Dhass PK. Aruna RM. Oxidative stress, interleukin-6 (IL-6) and atherogenic index of plasma in diabetic nephropathy. IJABPT. 2011; 2(2): 211-217.

9. Kalidhas P. Kanniyappan D. Gandhi K. Aruna RM. Coronary artery disease risk factors in type 2 diabetes mellitus with metabolic syndrome in the Urban South Indian population. Journal of Clinical and Diagnostic Research .2011; 5(3):516-518.

10. Arnett FC. Edworthy SM, Bloch DA, McShane DJ, Fries JF, Cooper NS et al. The American Rheumatism Association 1987. Revised Criteria for Classification of Rheumatoid Arthritis. Arth Rheum 1988; 31:315-24.

11. Dobiasova M. Frohlich J. The plasma parameter log (TG/ HDL-c) as an atherogenic index : correlation with lipoprotein particle size and esterification rate in apo B-lipoprotein- depleted plasma (FERNDL). Clin Biochem. 2001; 34: 583588.

12. Ledwozym A, Michalak J, Stepien A, Kadziolka A. The relationship between plasma triglycerides,cholesterol, total lipids and lipid peroxidation products during human atherosclerosis, Clin ChemActa, 1983; 155: 275-284.

13. McEntegart A, Capell HA, Creran D, Rumley A, Woodward M, Lowe GD. Cardiovascular risk factors, including 
thrombotic variables, in a population with rheumatoid arthritis. Rheumatology (Oxford). 2001;40:640-4.

14. Khovidhunkit W, Memon RA, Feingold KR, Grunfeld C. Infection and inflammation-induced proatherogenic changes of lipoproteins. J Infect Dis.2000; 181(suppl 3):S462-S472.

15. Georgiadis AN, Papavasiliou EC, Lourida ES, Alamanos Y, Kostara C, Tselepis AD et al. Atherogenic lipid profile is a feature characteristic of patients with early rheumatoid arthritis: effect of early treatment- a prospective, controlled study. Arthritis Res Ther. 2006; 8(3):R82.

16. Kowsalya R, Sreekantha, Vinod Chandran, Remya. Dyslipedemia with altered oxidant -antioxidant status in Rheumatoid arthritis. Int $\mathrm{J}$ of pharma and Biosciences.2011;2 (1): B424-B428.

17. Situnayake R, Kitas G: Dyslipidemia and rheumatoid arthritis. Ann Rheum Dis 1997, 56:341-342.

18. Dessein PH, Stanwix AE, Joffe BI: Cardiovascular risk in rheumatoid arthritis versus osteoarthritis: acute phase response related decreased insulin sensitivity and high-density lipoprotein cholesterol as well as clustering of metabolic syndrome features in rheumatoid arthritis. Arthritis Res 2002, 4:R5.

19. Munro R, Morrison E, McDonald AG, Hunter JA, Madhok R, Capell HA: Effect of disease modifying agents on the lipid profiles of patients with rheumatoid arthritis. Ann Rheum Dis 1997,56:374-377.

20. Zeinab M. A. R., Eiman A.A., Layla K.A., Noorhan K.S., Interleukin-33 in Iraqie ${ }^{\text {ee }}$ Rheumatoid Arthritis patients prone to Atherosclerosis. Medical Journal of Babylon-Vol. 10- No. 4 -2013. 